\title{
Empoderamiento en terapia radial: la experiencia de Radio Nueva Terapia
}

\section{Empowerment in radial therapy: the experience of Radio Nueva Terapia}

\author{
Camilo Salas Sandoval \\ Universidad de Chile
}

Constanza Román Avilés

Universidad de Chile

isabel.gonzalez@ug.uchile.cl

\author{
Isabel González Pastrán \\ Universidad de Chile
}

\section{Marta González Pastrán}

Universidad de Chile
Resumen

Abstract

Palabras

clave

Key

words
El artículo describe las principales características de Radio Nueva Terapia (RNT), proyecto comunicacional que se realiza en la comuna de Colina (Chile), y que consiste en la realización de programas radiales con personas con trastorno mental grave. La finalidad de estos es empoderar a sus destinatarios bajo la óptica de la psicología humanista y la psicología comunitaria. Para configurar esta perspectiva sobre el empoderamiento, el artículo enfatiza en el estigma internalizado inherente al etiquetamiento en salud mental como fenómeno que genera percepciones negativas sobre la propia identidad, así como sobre los beneficios que tienen las relaciones humanas "de calidad" entre las personas, y de cómo se circunscribe la radio, como herramienta comunicacional, en un contexto terapéutico como el de RNT, donde esta potencia la obtención de impacto fuera del contexto cercano de los participantes y sus familias.

The article describes the main features of Radio Nueva Terapia (RNT), a communication project developed in Colina (Chile), which consists on the implementation of radio shows conducted by people diagnosed with severe mental problems. The main purpose of this project is to empower the users of this program under the optics of different theoretical perspectives such as the humanistic psychology and the community psychology. To set this perspective about the empowerment, the article emphasizes, on one hand, the internalized stigma which is inherent to the labeling related with mental health issues, which generates negative perceptions of identity; and, on the other hand, on the benefits that "quality" human relationships have over people. To this extent, this article also highlights the importance of the radio, as a communication tool, in a therapeutic context such as RNT, where the radio is empowered by the impact it has over the immediate context of the participants and their families.

Radio nueva terapia, humanismo, empoderamiento, terapia radial.

Radio nueva terapia, humanism, empowerment, stigma.

○ Agradecimientos: A Red de Investigación en Salud Mental Sur 


\section{Introducción}

En términos generales, una terapia es una acción orientada a contribuir con el bienestar psicológico, social y biológico de una o más personas. Puesto que Radio Nueva Terapia (RNT) se ha orientado a colaborar con el bienestar de personas diagnosticadas con trastornos mentales tales como esquizofrenia y deficiencia mental, no es difícil considerar que se trata de una terapia. Ahora bien ¿en qué sentido se trataría de una nueva terapia? Cuando el proyecto comenzó, el año 2012, en Peñalolén, se realizó una conversación grupal para encontrar un nombre apropiado para el proyecto. Uno de los participantes mencionó que las actividades que aquí se realizaban eran "como un nuevo tipo de terapia" ¿Por qué él percibió esto? En general, las directrices y prácticas de la salud mental en Chile tienen un enfoque predominantemente farmacológico y de rehabilitación psicosocial, es decir, se enfocan en la remisión de síntomas de origen biológico, a la vez que buscan la inserción de los usuarios en el mundo de la educación y el trabajo. Además, el parecer del usuario del sistema de salud no tiene mayor injerencia en el desarrollo de la terapia, pues dichas prácticas que beneficiarían al usuario ya se encuentran definidas por el juicio de expertos y profesionales. De acuerdo a lo que se expone en este artículo, RNT se conforma como un espacio en el cual sus participantes pueden expresar sus intereses y desarrollarse como seres humanos, más allá de lo que prescriben los diagnósticos que orientan a las instituciones de salud mental. Así, no parece extraño que los participantes de esta iniciativa consideraran que el tipo de prácticas que se realizan en los talleres conforman una forma de terapia no tradicional, por lo que decidieron bautizar la iniciativa como Radio Nueva Terapia.

Los principales objetivos de RNT son: a) contribuir al empoderamiento colectivo e individual de personas con trastornos mentales severos, además de b) contribuir a la desestigmatización yaceptación de personasquehan sido diagnosticadascon enfermedades mentales. Si bien ambos objetivos se desarrollan desde la idea del empoderamiento, pues, como menciona Montero (2005), este incluye tanto la transformación de la organización o comunidad que incrementa su poder, así como del entorno que rodea a esa colectividad, se hará una división entre estos objetivos para así facilitar el entendimiento de los ámbitos de acción de RNT.

Para configurar esta perspectiva sobre el empoderamiento, el artículo comienza por relacionar distintas corrientes teóricas que permiten comprender el proyecto, tales como 
el humanismo y la psicología comunitaria. Se hace énfasis en el estigma internalizado inherente al etiquetamiento en salud mental como fenómeno que genera percepciones negativas sobre la propia identidad, así como sobre los beneficios que tienen las relaciones humanas "de calidad" entre las personas. La radio potencia, además, un impacto que va más allá del contexto cercano de los participantes y sus familias. Posteriormente, se describe la metodología de acción y el trabajo que se realiza dentro de los talleres, a través del desglose de los objetivos específicos de RNT y los conceptos teóricos que se transforman en quehaceres concretos al interior de las sesiones. Finalmente, se discutirá sobre las implicancias de este proyecto a futuro y las proyecciones que RNT tiene a corto y largo plazo.

\section{Consideraciones teóricas que sustentan Radio Nueva Terapia}

El empoderamiento (o potenciación) es un término central para la psicología comunitaria (Chacón et al., 1998) y es conceptualizado por Maritza Montero (2005) como "fortalecimiento", es decir:

El proceso mediante el cual los miembros de una comunidad (individuos interesados y grupos organizados) desarrollan conjuntamente capacidades y recursos para controlar su situación de vida, actuando de manera comprometida, consciente y crítica, para lograr la transformación de su entorno según sus necesidades y aspiraciones, transformándose al mismo tiempo a sí mismos (p. 72).

Para avanzar en la dirección multidimensional que contempla esta comprensión del empoderamiento, RNT hace hincapié en algunas consideraciones que pueden apreciarse en diferentes corrientes teóricas, configurándose como un conjunto de relaciones destinadas a producir formas de sentido individuales y grupales que contribuyan al fortalecimiento de sus destinatarios, pues se considera que: a) los seres humanos son productores de sentido y, de esta forma, se construyen a sí mismos. b) La producción de sentido es una actividad comunicativa y cultural, por lo que las relaciones con los "otros" y el mundo juegan un papel relevante en la elaboración del bienestar. c) La producción de sentido permite avanzar hacia un proceso de autodeterminación individual y colectiva. d) Todos los seres humanos son diferentes en cuanto a la producción de su propia subjetividad, por lo tanto, las formas de abordar los problemas subjetivos dependen de cada caso en particular y de las consideraciones que tiene cada vida sobre 
la dirección que debe tomar ${ }^{1}$. e) Todos los seres humanos son iguales en derechos, ya que la vida humana es valorable más allá de cualquier tipo de diferencia. Ello implica que todos merecen un trato respetuoso, afectivo, empático y, en buena medida, todos se benefician de relacionarse como seres valorados en su humanidad e individualidad.

Las personas se construyen a sí mismas produciendo sentido de diversas formas: a través del lenguaje y sus emociones, asimilándose en la cultura, siguiendo rutinas, relacionándose con otros y haciéndose una idea de lo que son sus vidas. Como diría Moffatt (2011) desde la psicoterapia existencial, el sentido es el hilo que teje la continuidad de la sucesión de presentes inconexos en los que se presenta a cada instante la existencia, dando a su realidad actual un papel dentro de una narración con la que se construye cada biografía. Es así como "hacer vida" produciendo sentido es una parte de la condición de la existencia humana. De esta manera, el bienestar y el malestar pasan a ser procesos particulares de producción de sentido (o "sinsentido"); formas que tienen las personas de experimentar sus vidas y contárselas a sí mismas².

La elaboración de sentido es un fenómeno experimentable desde la individualidad, pero es realizada a través de la comunicación entre los seres humanos y la cultura. En otras palabras, la mirada de los otros nos define, y con ellos se establece una negociación de sentido, a la vez que aprendemos la cultura relacionándonos con otras personas. "La vida es un largo diálogo con otros" (Moffatt, 2011, p. 4). De esta forma, el sentido de nuestras vidas está condicionado por las relaciones que hemos establecido con el mundo cultural y los otros.

Las consideraciones que tengamos sobre nosotros mismos, incluyendo el valor que nos entregamos y las creencias sobre nuestras capacidades, están influenciadas por nuestra historia de relaciones con los otros y el mundo cultural. Los estereotipos y discriminaciones

1 Con excepción de (4), todos los puntos serán desarrollados. Respecto a (4), basta mencionar que la consideración sobre la diversidad humana es muy relevante en algunos enfoques de salud mental. Por ejemplo, el "diálogo abierto", el abordaje de la psicosis que ha tenido los mejores resultados en el mundo desarrollado, tiene su origen en el "tratamiento adaptado a las necesidades", el cual se desarrolló desde 1969 en Finlandia. En este enfoque se consideraba que las personas con psicosis eran heterogéneas, por lo tanto, a algunas les podía servir un tipo de intervención -como el tratamiento farmacológico-mientras que a otras no. No se concentraban en la remisión de síntomas -objetivo básico en el modelo médico en salud mental-sino que preguntaban a las personas sobre su vida, sobre sus éxitos y su vida cotidiana, en otras palabras, se escuchaba a los pacientes y se les ayudaba a construir narrativas sobre si mismas (Withaker, 2010).

2 En los origenes de las perspectivas existenciales de la psicología, se considera que el ser humano, aun en las situaciones más difíciles, puede sostenerse a sí mismo aferrándose a alguna clase de sentido, tal como lo desarrolla Victor Frankl (1991), quien, a raíz de su experiencia en un campo de concentración nazi, concluye que el sentido es la forma primordial con la que el ser humano puede hacerle frente al sufrimiento y conseguir bienestar mental. 
que otros ejercen sobre las personas con algún diagnóstico psíquico -el llamado "estigma"atentan contra la valorización que estas personas realizan sobre sí mismas. A ello se le llama estigma internalizado y es definido por Corrigan (1998) como "la devaluación, vergüenza, secrecía y retraimiento consecuencia de aplicar los estereotipos negativos a sí mismo". Así, el auto-estigma se asocia con baja autoestima y esperanza (Livingston y Boyd, 2010), las cuales, a su vez, pueden ser leídas como formas particulares de producir sentido sobre el "yo" y sobre el propio futuro, correspondientemente.

Por otra parte, el estigma internalizado genera la creencia que las personas no son capaces de hacer lo que se proponen, es decir, disminuyen su autoeficacia (Brohan et al., 2010). Las creencias sobre las propias capacidades son importantes para que las personas puedan dirigir sus vidas: para alcanzar algo, es necesario creer que es posible. Por esto la autoeficacia es un elemento importante del empoderamiento (Musitu, 2004). En consecuencia, la posibilidad de guiar la propia subjetividad es relevante para muchas corrientes de la psicología y el bienestar subjetivo en general, y para el tratamiento de los llamados trastornos mentales graves ${ }^{3}$ en particular. Se ha subrayado la importancia de este tipo de factores subjetivos en los procesos de recuperación de personas con este tipo de malestares (Garay et al., 2014).

El incremento de la capacidad de autodirigir la propia vida, desde el ámbito de la producción de sentido, es relevante para el desarrollo de los seres humanos en general, más allá de la etiqueta o diagnóstico que porten ${ }^{4}$. Corrientes teóricas como la psicología positiva, el mindfulness y la terapia de aceptación y compromiso dan cuenta de ello (Kashdan y Ciarrochi, 2014), ya que muestran que, independiente de si las personas tienen o no un diagnóstico, estas son capaces de aprender a conducir su bienestar controlando la forma en la que producen sentido (a través del manejo de las emociones, la meditación, la autocompasión, la concentración en experiencias positivas, etc.). Lo que está en juego en el desarrollo de una persona, más allá de la remisión de síntomas de una enfermedad, es la expansión del potencial humano.

3 El enfoque de recuperación (recovery) es un ejemplo importante de las tendencias que ponen la autodeterminación como un factor primordial del mejoramiento en salud mental. La recuperación se encuentra en la dimensión existencial de los afectados (Whitley y Drake, 2010) y se define como un "viaje" subjetivo a través del cual las personas pueden reformular el sentido de sus vidas y autodirigir su propio bienestar (SAMHSA, 2004). Se fomenta la esperanza y la creación de un sentido de poder interno (Spaniol et. al., 1994) que le permite al usuario establecer un compromiso con su propio proceso de mejoramiento. En estudios longitudinales, se ha visto que factores de índole subjetivo, como la autoeficacia, son las claves primordiales en quienes atraviesan un camino óptimo de recuperación (Harding, Brooks, Ashikaga et. al., 1987; Jobe y Harrow, 2005).

4 Puntos de vista rigurosos y recientes descartan la existencia de enfermedades mentales en general, y se cuestiona la existencia y pertinencia de constructos como la esquizofrenia (Read, 2006) y el déficit atencional (García et al., 2014). 
Otro aspecto importante a considerar es la idea que el trato que tiene el profesional con el "consultante" es más relevante que la técnica utilizada. Tras analizar las grabaciones de diversos psicoterapeutas, Carl Rogers (1988), uno de los fundadores del enfoque humanista en psicología, resaltó que lo que daba efectividad a la psicoterapia eran elementos de la relación terapeuta-paciente, tales como la aceptación positiva incondicional, la calidez y la empatía. De acuerdo a lo expuesto anteriormente, esta observación de Rogers puede ser más pertinente aún para la interacción con personas que han sido etiquetadas como discapacitadas por problemas de salud mental. En este contexto, la calidez y horizontalidad de las relaciones humanas - relevantes en sí para la psicoterapia y para las relaciones en general- puede marcar una diferencia relevante en torno a las consideraciones que una población vulnerable tiene sobre sí misma.

Como ya se ha dejado ver, la perspectiva de este artículo es que el desarrollo del potencial humano está mediado por la cultura y los procesos comunicativos de los cuales las personas forman parte. A nivel colectivo, el empoderamiento implica relaciones de respeto, aceptación mutua, el compromiso con ciertos valores compartidos, así como otra serie de actividades que brindan la oportunidad para el desarrollo personal de cada miembro de la organización. Cuando una organización desarrolla prácticas y valores para lograr objetivos comunes, y cuando se fomenta la "calidad" de las relaciones humanas, se puede generar un proceso de autodeterminación colectiva. Además, es necesario que se desarrollen diálogos entre todos aquellos que son parte de la conformación de prejuicios para desestigmatizar a las personas con diagnósticos en salud mental. Es aquí donde pueden jugar un papel importante los medios de comunicación masiva, ya que estas instituciones pueden propiciar transformaciones en las producciones de sentido que forman parte de la cultura. "La radio justamente funciona como escenario, como plataforma de encuentro que ayuda a modificar miradas, por lo tanto realidades" (Olivera 2014 p.1). Así, las transmisiones en una radio local tienen la oportunidad de tensionar y cambiar la percepción que tienen sus auditores sobre quienes son diferentes. Además, se habilita un vínculo entre los usuarios y la comunidad, dándole la posibilidad a esta última de escuchar, reflexionar y participar en el proceso terapéutico de los usuarios, al formar parte de un espacio donde los participantes pueden ser escuchados y convertirse en seres humanos opinantes y críticos.

Considerando que, históricamente, se han vulnerado los Derechos Humanos de los discapacitados y de las personas clasificadas por la psiquiatría, al no considerar su 
dignidad o autonomía, ni sus necesidades económicas o sociales (el estigma es una parte importante de la vulneración), es que la distinción de un proyecto como RNT está dada por hacer énfasis en que los problemas de salud mental deben ser abordados por toda la sociedad, y no sólo por quienes los padecen. La utilización de un medio de alcance masivo como la radio considera que el malestar y el bienestar se experimentan individualmente, pero que se encuentran mediados por producciones colectivas.

\section{El desarrollo de los objetivos de Radio Nueva Terapia en la práctica}

RNT surgió en agosto del año 2012, a través de la inquietud de un grupo de jóvenes universitarios que querían trabajar la esquizofrenia desde una perspectiva interdisciplinaria e innovadora, teniendo en cuenta la experiencia de Radio La Colifata en Buenos Aires. El proyecto ha estado presente en los Centros Comunitarios de Salud Mental (COSAM) de Peñalolén, Lampa y Colina. En la actualidad, este se lleva a cabo en la comuna de Colina con participantes de la red de discapacidad de la localidad, integrando a jóvenes de COSAM, Coanil, Colegio Unamkalem, la agrupación Jóvenes con Esperanza y a quienes han adherido por iniciativa propia. Con ellos se realizan talleres semanales de radiodifusión en el Centro Cultural de la comuna. Además, se transmite un programa semanal, bautizado por los mismos participantes como Psiquiatricool, a través del medio local Radio Sembrador, que se difunde en la comuna y por internet.

Para esquematizar cómo es que la teoría que está a la base de RNT y sus objetivos principales -empoderar y desestigmatizar-son aplicados en los talleres de radiodifusión y radioteatro, es posible agrupar las experiencias de la iniciativa en seis objetivos específicos que se expondrán con detalle, señalando las actividades realizadas de acuerdo a estas metas y acompañado de testimonios que plasman el progreso de las prácticas:

3.1. Dialogar sobre el estigma internalizado entre los participantes para superar, en conjunto, las características negativas que inspiran los etiquetamientos y la discriminación.

Como es de suponer, los participantes de RNT han sido discriminados de distintas formas a lo largo de sus vidas. Por ejemplo, Magaly $(21)^{5}$, con diagnóstico de depresión y movilidad reducida, le ha narrado a un monitor lo siguiente: "Me hace sentir mal cuando me discriminan, cuando me pisotean. Un día mi mejor amigo se pone a llorar para una

5 Los nombres utilizados son seudónimos, aunque se ha conservado el género y la edad. 
Teletón y me dice que no puede bailar con una discapacitada. Ahí me quebré y me puse a llorar. Nos pusimos a llorar los dos". Así mismo, Jasmín (50), diagnosticada de esquizofrenia, cuenta -en una transmisión en vivo en Radio Sembrador- que no podía comprender por qué las personas de su barrio se comportaban de forma agresiva con ella. Avanzado su relato, recordó que la agredieron físicamente fuera de su casa, sin ninguna razón clara.

Para superar los problemas asociados a cómo los participantes internalizan la discriminación, un primer paso posible es la narración de experiencias. Por medio del diálogo, es posible resignificar algunas vivencias dolorosas. Por ejemplo, cuando Jazmín contó su relato, un compañero le dijo que el comportamiento de sus vecinos era fruto de la ignorancia. También agregó que ella no tenía "nada de malo", a la vez que otros compañeros la animaron con sus propias opiniones.

Los participantes de RNT han dialogado sobre el estigma en distintas instancias, tanto en los talleres como en las transmisiones radiales y algunos eventos para la comunidad. Incluso se habló del tema en la primera visita a Radio Sembrador. Ello quedó registrado en una nota realizada por el noticiero de Canal 13 -medio televisivo de alcance nacional-que asistió a la actividad. En la nota, hay un diálogo entre dos participantes jóvenes que, en ese momento, son transmitidas por Radio Sembrador:

Andrea (31): La gente se burla...

Vilma (21): ¿De qué se burlan, de ti?

Andrea: De mi forma de ser. Mucha gente cree que yo soy tonta...

Vilma: ¿Y tú creí que para ti, para ti, tu soi tonta?

Andrea: No, por supuesto que no, ustedes ya me conocen, saben mi personalidad, no creo que sea...

Vilma: Entonces, que te de lo mismo lo que creen los demás.

3.2 Se fomenta un espacio de reconocimiento, respeto y aprecio que permita que los miembros del grupo se auto-valoren y se vean como seres humanos iguales en dignidad.

La importancia del trato con y entre los participantes puede ser incluso más significativa que la metodología de trabajo que se lleva a cabo. Un espacio básico de resistencia ante el estigma en salud mental, y ante las formas de producción de sentido negativas, es el conjunto de relaciones comunicativas con las que los grupos pueden brindarse apoyo y producir formas de sentido alternativas. El dominio sobre la propia subjetividad puede 
ser fomentado desde los afectos y emociones que surgen en los procesos comunicativos, promoviendo el desarrollo de micro-culturas de resistencia ante formas hegemónicas de producción de significado.

En los talleres se han realizado actividades orientadas específicamente a establecer reglas de convivencia y respeto, resolución de conflictos, y valoración de cada uno de los integrantes. Por ejemplo, durante agosto del 2014, en los talleres de Colina, se realizó una actividad llamada "Las reglas del grupo", donde los participantes crearon reglas para sí mismos. Algunas de estas normas eran: "No nos debemos tratar mal entre nosotros", "Si estamos en contra de la discriminación, no podemos discriminarnos entre nosotros", "Seamos todos amigos y hermanos", "Seamos una familia". A partir de estas actividades, Mario (27) ha dicho que "acá en la radio me siento aceptado y no quiero salirme del Taller porque aquí tengo amigos y amigas, todos son mis amigos aquí". A su vez, Franco (26), diagnosticado de esquizofrenia, afirma "me llevo bien con los compañeros, nos respetamos y me he sentido súper cómodo. Los monitores son amigables y buena onda". Y en el testimonio de Juan Cristobal(45), diagnosticado de epilepsia y déficit intelectual, se lee que "la gente me quiere mucho, aquí me quieren mucho. Todos nos queremos mucho. No hay ningún maltrato".

Por último, es necesario apuntar que los talleres dejan lugar para la improvisación y el juego, manteniendo un trato informal y abierto, y evitando actitudes autoritarias.

3.3. Se fomenta su participación e incidencia en el devenir del proyecto, lo que implica una valoración de ellos mismos como personas capaces de organizarse, pensar y decidir.

La posibilidad de que los participantes puedan tomar decisiones sobre las actividades que realizan, es otra de los objetivos de RNT. En este sentido, la terapia que propone este proyecto no está decidida de antemano, sino que ellos tienen injerencia sobre las decisiones que aquí se toman. Si bien claramente hay un marco conceptual base sobre el cual se trabaja, ellos son finalmente los que dirimen sobre temas como los contenidos radiales, las secciones del programa, los roles de los participantes, la organización de eventos de radio abierta, la definición de temas a tratar en cada sesión y principalmente la evaluación de las transmisiones radiales junto a las actividades realizadas semanalmente. Esto se genera desde la idea de un espacio de reconocimiento y respeto, pero, principalmente, como un espacio donde ellos son jueces y espectadores de su propio accionar, donde 
se puedan responsabilizar individual y colectivamente de las decisiones que toman. En base a esto, Cristobal B. (38) diagnosticado con esquizofrenia, comenta su experiencia y reflexiona sobre cómo la radio "me ha servido bastante. He podido hacer cosas que no hacía hace tiempo, he vuelto a escribir. Yo lo he visto como un espacio donde tratamos de hacer algo distinto a lo que hacía antes, mantener la mente ocupada y que no se me adormezca. Ayuda en el vocabulario, ayuda a distraerse, a no estar tan encerrado en sus propios problemas. Muchas cosas ayudan a las personas, pero no lo saben hasta que se dan cuenta". En este último punto radica la importancia de la incidencia de los participantes en el devenir del proyecto, pues el tener la posibilidad de valorarse como seres opinantes en un entorno que está considerando su voz (pues hay otros escuchando sus programas radiales) les permite salir del espacio cotidiano y en palabras de Cristóbal "no estar tan encerrado en sus propios problemas".

3.4. Se procura que adquieran herramientas y competencias expresivas y comunicacionales con las que puedan sentirse más satisfechos con su forma de relacionarse con los otros y con el mundo.

Uno de los ejes centrales desde el inicio del proyecto ha sido el potenciar habilidades expresivas y comunicacionales en los participantes. Con esto, pueden revalorar las formas de relacionarse y re-entender no solo su propia forma de expresarse, sino también la de los otros que habitan su entorno, lo cual les permite sentir empatía con ellos mismos y con el resto. Frente a esto, Claudio Acevedo, terapeuta ocupacional en el COSAM de Colina, comenta en una nota realizada para el diario Hoy $x$ Hoy una situación vivenciada con uno de los participantes: "recuerdo uno de los casos, del cual no puedo dar el nombre, en que asistí a una sesión y vi discutiendo al joven en cuestión en un radioteatro. Me impresionó mucho verlo participar, opinar, aportar con ideas de una manera bastante activa, con un desarrollo del lenguaje que él no tenía previamente. Tenía la capacidad de entender que si otro habla, tenía que esperar su turno. Eso fue fantástico" (p.8). La idea es entonces que ellos adquieran herramientas que sólo se pueden aprender desde la práctica.

También surge otro aspecto importante que el trabajo en radio permite: hacer consciente procesos que muchas veces se realizan de manera automática. En general, las personas no procesan de manera consciente la estructura de las conversaciones (apertura, tiempos de pausa, etc) u otros elementos relacionados a estas (volúmen, organización de ideas, etc), por lo tanto el tener la posibilidad de vincular estos a un ámbito concreto de acción les 
permite pensar en estos elementos al tiempo que los ponen en práctica. En relación a esto, Valentín (49) no vidente y diagnosticado de trastorno bipolar, comenta "me ha parecido bien interesante (la experiencia), porque a través del trabajo de la radio aprendemos a expresarnos mejor. El hecho de que de repente uno esté en la radio y tenga que hablar sobre un tema específico, lo obliga a estar pensando y locuteando al mismo tiempo".

3.5. Se fomenta la articulación de relaciones interpersonales y redes sociales que permitan incrementar el bienestar (fomentando relaciones de respeto, la amistad entre los compañeros del grupo, su participación en distintas instancias sociales y el desarrollo de sus competencias comunicativas por práctica radial).

Este objetivo se aborda desde dos perspectivas distintas. Por una parte, está el trabajo al interior del proyecto, mientras que, por otra, se encuentra el trabajo de vincular el proyecto con otras entidades o instituciones. El primer enfoque se lleva a cabo a través de diversas actividades que toman el diálogo como eje central. Es así como en cada taller se genera un momento de esparcimiento (la colación) donde cada uno es libre de hacer lo que quiera, manteniendo el respeto por los otros. Muchas veces, en este espacio, los participantes cantan, bailan o simplemente conversan sobre cosas externas al taller, es decir, situaciones familiares que hayan acontecido, cosas que hayan vivenciado en su entorno cercano, etc. Asimismo, y de manera natural, se han ido generando diversos vínculos en el taller, se han formado diversas relaciones de pareja y de amistad. Estos vínculos han surgido principalmente desde el reconocer en los otros a un par. Debido a esto, muchos buscan la inclusión y la unión grupal. Por ejemplo, Marcelo (39), quien en un inicio siempre se mantenía más distante del resto del grupo, ha ido integrándose lentamente gracias a que César (45) constantemente lo invita a participar de las conversaciones o consulta su opinión cuando se organiza la pauta radial. Con esto, César le otorga un valor a la opinión de Marcelo, lo cual permite que este último pueda participar cada vez más en las actividades. Lo mismo ocurre con dos participantes con movilidad reducida, Magaly y María Inés. Ambas son asistidas por todos sus compañeros al momento de subir o bajar escaleras, o bien en los trayectos desde sus casas al lugar de realización de los talleres (Centro Cultural de Colina). En base a esta misma idea, Valentín comenta "en lo personal, yo estaba bien solo y lo de compartir con otras personas me ha servido mucho para relacionarme y expresarme".

El segundo enfoque de formación de redes, es la vinculación del proyecto con el resto de la comunidad $u$ actores específicos. Esto se realiza a través del trabajo radial, así como del 
trabajo en conjunto con otras instituciones que permitan ampliar las redes sociales de los participantes más allá de las que generen al interior del taller. Es por esto que, desde el inicio del proyecto en la comuna de Colina, RNT ha estado en contacto con la Red de Discapacidad de la comuna, donde se encuentran diversas organizaciones tanto de familiares como de usuarios de otras iniciativas.

3.6. Se llevan a cabo estrategias conjuntas para aumentar el poder del proyecto, incrementando los recursos con los que se cuenta, sus vínculos, los espacios en los que se participa, etc. Ello implica que los miembros de la organización puedan sentir que forman de un proyecto que avanza y es valorable.

Los profesionales y participantes comparten el objetivo de difundir los programas radiales y posicionar el espacio en la comunidad. Con esta finalidad se buscó la inclusión de RNT en Radio Sembrador y el posterior lanzamiento del espacio radial Psiquiatricool en Colina. Para esto, se han gestionado diversas notas para medio locales (TV Colina canal 6, Radio Cumbres, Radio Colina) y para otros de alcance nacional (C13, 13C y Hoy x Hoy). El proyecto también es difundido en internet, a través del fan page de RNT en Facebook y del canal Youtube de la radio.

Asimismo, se ha participado en diversos eventos donde los participantes van a exponer sus experiencia en la radio, tales como la V Feria de la Discapacidad, realizada en 2013; la Feria por la Inclusión en Salud Mental, también realizada en 2013; el evento Alternativas a la Psiquiatría en 2015 y el Ciclo de Encuentros Alternativas a la Psiquiatría en 2015. Lo que se persigue con estas actividades no es sólo visibilizar el proyecto y con esto ampliar la cobertura del programa radial, sino también que los participantes puedan sentir que su trabajo está siendo valorado socialmente y que ellos también son capaces de aportar y generar conocimiento en otros.

A partir de esto, Rodrigo (54), quien fuera diagnosticado con esquizofrenia paranoide, afirma que "yo encuentro que la radio me ha ayudado bastante, por eso me gusta, y también creo que nosotros hemos ayudado a otra gente acá. Lo importante es que ponemos un granito de arena, toda la gente que viene $y / o$ escucha la radio, como los estudiantes, $u$ otras personas... porque yo creo que desde mi realidad yo puedo enseñar cosas. A la gente que viene, la tratamos bien y feliz porque lo pasamos bien, nos llevamos bien con ellos. Nosotros nos sentimos bien como nos atienden acá y eso se agradece". Lo que propone Rodrigo con 
este comentario es precisamente uno de los objetivos discutidos anteriormente, la idea de empoderamiento, pues los participantes dejan de ser meros receptores de información, sino que son capaces de articular discursos y proponer soluciones a otras personas que pueden estar o no en su entorno cercano. Se convierten entonces en artífices de su propia realidad y por ende en miembros activos de la comunidad en la que viven.

\section{Discusión / conclusiones:}

RNT aborda aspectos que no están lo suficientemente considerados en las políticas de salud mental chilenas o en los tratamientos convencionales para personas diagnosticadas con esquizofrenia u otros problemas de salud mental. En síntesis, se considera que un aspecto central de la producción de bienestar, en y más allá del campo de la salud mental, se encuentra en la generación de sentido a través de procesos comunicativos que permitan abrir espacios culturales en los que se fomenten las capacidades de autodeterminación y se diluya la discriminación y las formas de sentido común que inspiran una elaboración negativa de la propia identidad. Al contrario de los enfoques que buscan situar y tratar la problemática psíquica, de forma casi exclusiva, al interior del individuo, RNT asume que las experiencias de bienestar y malestar están mediadas por la cultura y la relación con los otros, lo que implica que el desarrollo del ser humano y la superación del sufrimiento son tareas que comprometen a toda la sociedad. Así, los medios de comunicación masiva, en tanto que dispositivos privilegiados para coordinar las comunicaciones y la elaboración de sentido a gran escala, tienen el potencial de ser herramientas primordiales para la producción de una cultura para el desarrollo del bienestar psíquico y, más particularmente, para el fomento de la aceptación, el respeto y la valoración de la diferencia, tanto en el caso de los trastornos mentales severos como en cualquier otro (diversidad sexual, económica, étnica, etc ${ }^{6}$.).

En este artículo, se ha profundizado sobre cómo la generación de formas de sociabilidad a pequeña escala (o "micro-culturas") podrían incrementar el bienestar de los individuos pertenecientes a un grupo, a través del fomento de algunas líneas como el diálogo respecto de la discriminación, el respeto y la valoración entre los miembros del grupo y el incremento del poder colectivo, facilitando el empoderamiento o autodeterminación de los participantes de RNT. Queda aún pendiente cómo es que la ampliación de este tipo de líneas, a nivel comunal o nacional, podría favorecer la autodeterminación de las personas,

6 La discriminación en todas estas áreas también tiene efectos psíquicos, aunque no sean motivo de diagnóstico. 
particularmente, a través de la disolución del estigma asociado al diagnóstico psiquiátrico, que dificulta las relaciones personales, así como el desempeño en el trabajo y el mundo educativo. Es necesario implementar mecanismos para evaluar el impacto de la difusión del proyecto a nivel local, así como incrementar los esfuerzos en este sentido.

Por otro lado, es necesario realizar evaluaciones que permitan establecer en qué medida este tipo de intervención permite a sus participantes la real incorporación en su comunidad, así como diluir el estigma asociado al diagnóstico psiquiátrico. Para esto, actualmente se está llevando a cabo una investigación de carácter contrastivo que ahonda en estas diferencias y permite establecer nuevas metas y parámetros para el desarrollo del proyecto. A pesar de que todavía no se cuenten con datos concretos sobre el impacto de este proyecto, y como ya se ha estado mencionando a lo largo de este artículo, si es posible observar avances y modificaciones en ciertos comportamientos de los participantes. Desde aquí, parte del personal del COSAM Colina, ha hecho hincapié en los avances que ha visto en los participantes: "he visto mejoras increíbles con el tema comunicacional, principalmente la expresión hablada del lenguaje, en lo que se refiere a las habilidades sociales, a la capacidad de interactuar con otros y expresar sentimientos. Tienen la posibilidad de sentirse partícipes de la comunidad y eso les genera una sensación de pertenecer, les eleva la autoestima y dejan de sentir que son una carga, se dan cuenta que tienen opinión y que son un aporte a la sociedad. Muchas veces se ven más afectados en sus relaciones interpersonales. El fármaco no es la solución, un usuario puede estar estable pero hay que hacerlo partícipe de otros contextos dentro de su familia, comunidad y sus amistades" (Hoy X Hoy 2015).

\section{Referencias}

Brohan, Elaine; Gauci, Dolores; Sartorious, Norman; Thornicroft, Graham. (September 2010). Self-stigma, empowerment and perceived discrimination among people with bipolar disorder or depression in 13 European countries: The GAMIAN-Europe study. Journal of Affective Disorders, 129, pp. 56-63. http:// www.revistasaludpublica.uchile.cl/index.php/RCSP/issue/view/2589

Chacón, F. y García, M. (1998). Modelos teóricos en psicología comunitaria. En González, M. (Coord.). Psicología comunitaria: fundamentos y aplicaciones (31-47). España: Síntesis.

Corrigan, P. W. y Watson, A. C. (2002). Understanding the Impact of Stigma on People with Mental Illness. World Psychiatry, 1, 1, pp. 16-20. http://www.ncbi.nlm.nih.gov/pmc/articles/PMC1489832/

Frankl, V. (1991). El Hombre en Busca de Sentido. Barcelona: Herder. http://markeythink.files.wordpress. com/2011/04/el_hombre_en_busca_de_sentido_viktor_frankl.pdf 
Garay Arstegui, Mariasun; Pousa Rodriguez, Verónica y Perez Cabeza, Lucia. La relación entre la percepción subjetiva del funcionamiento cognitivo y el autoestigma con la experiencia de recuperación de las personas con enfermedad mental grave. Rev. Asoc. Esp. Neuropsiq. [online]. 2014, vol.34, n.123, pp. 459-475. ISSN 0211-5735.

García, F.; González, H. y Pérez, M. (2014). Volviendo a la Normalidad: la invención del TDAH y el trastorno bipolar infantil. Barcelona: Alianza Editorial.

Livingston, J. D., \& Boyd, J. E.. (2010). Correlates and consequences of internalized stigma for people living with mental illness: A systematic review and meta-analysis. Social Sciences Medicine, 71, pp. 2150-2161. http://scielo.isciii.es/pdf/neuropsiq/v28n1/v28n1a04.pdf

Moffatt, A. (2011). Psicoterapia Existencial. Buenos Aires: Esperanza.

Montero, Maritza (2005). Teoría y Práctica de la Psicología Comunitaria. Buenos Aires: Ed. Paidós.

Musitu, G. y Buelga, S. (2004). Desarrollo Comunitario y Potenciación. En Musitu, G.; Herrero, J:; Cantera, L. y Montenegro, M. (Eds.), Introducción a la Psicología Comunitaria (pp. 167-195). Barcelona: UOC

SAMHSA (2004). Declaración nacional de consenso sobre la recuperación en salud mental. http:// store.samhsa.gov/shin/content/SMA08-4129S/SMA08-4129S.pdf

Read, J:; Mosher, L. y Bentall, R. (eds.). (2006). Modelos de locura. Aproximaciones psicológicas, sociales y biológicas a la esquizofrenia. Barcelona: Herder.

Rogers, C. (1988). El proceso de convertirse en persona. México: Paidós.

Sartre, J-P. (2007). El Existencialismo es un Humanismo. Barcelona: EDHASA.

Vargas-Téllez, J. y Dorony-Saturno, L. (2013). Psicoterapia y acompañamiento: Un análisis conceptual desde el humanismo y la teoría de la autodeterminación. Revista de Psicología GEPU, 4 (2), 142 153. http://revistadepsicologiagepu.es.t//Psicoterapia-y-Acompa\%F1amiento-d--Un-An\%E1lisisConceptual-desde-el-Humanismo-y-la-Teor\%EDa-de-la-Autodeterminaci\%F3n.htm

Whitaker, R. (2010). Anatomy of an Epidemic: Magic Bullets, Psychiatric Drugs, and the Astonishing Rise of Mental IIIness in America. EE.UU.: Crown Publishing Group

Whitley R. y Drake R. (2010). Recovery: A dimensional approach. Psychiatric Service, 61, 1248-1250.

Zimmerman, P. (2000). Empowerment Theory: Psychological, Organizational and Community Levéis of Analysis. En J. Rappaport y E. Seidman (Eds.), Handbook of Community Psychology (pp. 43-64). Nueva York: Kluwer Academic Plenum. 